\title{
BERNOULLI SHIFTS ARE DETERMINED BY THEIR FACTOR ALGEBRAS ${ }^{1}$
}

\author{
PAUL C. SHIELDS
}

We shall show that, if $S$ is an invertible measure-preserving transformation of the unit interval with the same factor algebras as a Bernoulli shift $T$, then $S$ is isomorphic to $T$. This answers affirmatively a conjecture by G.-C. Rota in 1968.

Definitions. A transformation $T$ is an invertible measure-preserving transformation of the unit interval $X$ with Lebesgue sets $\Sigma$ and Lebesgue measure $\mu . T$ is called ergodic if there are no $T$-invariant sets $A \in \Sigma$ with $0<\mu(A)<1$. A factor algebra of $T$ is a complete $\sigma$-algebra $\Sigma_{1} \subseteq \Sigma$ such that $T \Sigma_{1}=T^{-1} \Sigma_{1}=\Sigma_{1}$. A partition $P$ is a countable disjoint collection of measureble sets whose union is $X$. The join of $P$ and $Q$ is $P \vee Q=$ $\{p \cap q \mid p \in P, q \in Q\}$ and $\bigvee_{k}^{n} T^{i} P=T^{k} P \vee T^{k+1} P \vee \cdots \vee T^{n} P$. Also, $\bigvee_{-\infty}^{\infty} T^{i} P$ denotes the smallest factor algebra containing $P$. $P$ is a generator for $T$ if $\bigvee_{-\infty}^{\infty} T^{i} P=\Sigma$ and $\left\{T^{i} P\right\}$ is independent if, for each $n>0, P$ is independent of $\bigvee_{1}^{n} T^{i} P . T$ is a Bernoulli shift if it has a generator $P$ such that $\left\{T^{i} P\right\}$ is independent.

The entropy $H(P)=-\sum_{p \in P} \mu(p) \log \mu(p)$, the relative entropy

$$
H(T, P)=\lim n^{-1} H\left(\bigvee_{1}^{n} T^{i} P\right) \text { and } H(T)=\sup _{p} H(T, P) .
$$

We have

(1) $H(T, P) \leqq H(P)$ with equality iff $\left\{T^{i} P\right\}$ is independent.

(2) If $P$ is a generator for $T$, then $H(T)=H(T, P)$.

(3) If $T$ is ergodic and $\varepsilon>0$, there is a generator $P$ such that $H(P) \leqq$ $H(T)+\varepsilon$.

The reader is referred to [3] for details about entropy. The result (3) is due to Rohlin and is the primary tool in our proof of the following theorem.

THEOREM. If $S$ has the same factor algebras as a Bernoulli shift $T$, then $S$ is isomorphic to $T$.

Received by the editors January 11, 1973 and, in revised form, April 9, 1973.

AMS (MOS) subject classifications (1970). Primary 28A65.

Key words and phrases. Bernoulli shift, factor algebra, entropy.

${ }^{1}$ This work is partially supported by NSF Grant GP 33581 X. 
Proof. We begin by making two elementary observations:

(4) $S$ is ergodic.

(5) $\bigvee_{-\infty}^{\infty} T^{i} P=\bigvee_{-\infty}^{\infty} S^{i} P$ for any $P$.

To prove (4), note that, if $S A=A$, then $\{\Phi, A, X-A, X\}$ is a factor algebra of $S$, hence of $T$. Since $T^{2}$ is ergodic, this implies that $\mu(A)$ is 0 or 1 . The result (5) follows from the fact that $P$ is contained in the factor algebra $\bigvee_{-\infty}^{\infty} T^{i} P$ of $T$, hence so is $\bigvee_{-\infty}^{\infty} S^{i} P$.

We now prove

(6) $H(S)=H(T)$.

First use (3) to choose a generator $P$ for $S$ such that $H(P) \leqq H(S)+\varepsilon$. $P$ must be a generator for $T$, from (5), and (1) and (2) give

$$
H(T) \leqq H(P) \leqq H(S)+\varepsilon .
$$

Now interchange the roles of $S$ and $T$ to obtain (6).

To complete the proof of the theorem, choose a generator $P$ for $T$ such that $\left\{T^{i} P\right\}$ is independent. We then have $H(T)=H(T, P)=H(P)$, and $P$ is a generator for $S$. Therefore,

$$
H(S)=H(S, P) \leqq H(P)=H(T)=H(S),
$$

so (1) implies that $\left\{S^{i} P\right\}$ is independent.

REMARKS. 1. The result (6) only uses the fact that $T^{2}$ is ergodic and can be easily proved assuming only that $T$ is ergodic.

2. Any two irrational rotations of the unit circle have the same factor algebras [1].

3. If $T$ is a $K$-automorphism [3], then $S$ must also be a $K$-automorphism. However, since there is a $K$-automorphism $T$ which is not isomorphic to $T^{-1}$ (see [2]), the theorem is false for $K$-automorphisms.

\section{REFERENCES}

1. R. L. Adler, Invariant and reducing-subalgebras of measure preserving transformations, Trans. Amer. Math. Soc. 110 (1964), 350-360. MR 28 \#183.

2. D. S. Ornstein and P. Shields, An uncountable family of K-automorphisms, Advances in Math. 10 (1973), 63-88.

3. V. A. Rohlin, Lectures on the entropy theory of transformations with invariant measure, Uspehi Mat. Nauk 22 (1967), no. 5, (137), 3-56=Russian Math. Surveys 22 (1967), no. 5, 1-52. MR 36 \#349.

Departmfint of Mathematics, Stanford University, Stanford, California 94305

Current address: Department of Mathematics, University of Warwick, Coventry, England 